\title{
The Essences and Stabilities of Substances
}

\author{
Yu Han (Chinese name 韩雨) \\ identitycard number:211402198310140019 \\ E-mail: hy301hos@163.com \\ Telephone:15011578199
}

The substances are expressions of dynamic structures with interactions.No matter whatMacro-materials or Micro-materials both interact with external dynamic structures. In fact,the natures and stabilities of substances are maintained by their inner dynamic structures interacting with external dynamic structures.The philosophical essence of stabilities of substances maintained is that inner systems interact with external systems. Besides external systems inner systems interact with other systems. Inner dynamic structures interact with external dynamic structures by kinds of ways. For example, the inner dynamic structures exchange dynamic structures with external dynamic structures. (1)

In a narrow sence,inner systems interacting with external systems do not include other systems. The generalized inner systems interacting with external systems include other systems.

For instance, the essences、stabilities and so on of electrons、 neutrons and protons and so on are maintained by the way that Inner dynamic structures interact with external dynamic structures.In fact,the essences、stabilities and so on of molecules、 inorganics、organics、cells、 organisms and so on are maintained by the way that Inner dynamic structures interact with external dynamic structures.

In fact, natures of substances are maintained by the way that Inner dynamic structures interact with external dynamic structures.

For example, the natures of resistances are maintained by the way that Inner dynamic structures interact with external dynamic structures.

In fact substances and things and so on are maintained by the way that Inner dynamic structures interact with external dynamic structures (and other systems).

1.The Theory of Changes in Extreme Conditions. The Properties and Trends of Changes and Movements. The Theory of the Dynamic Structures. Volume 4 Issue 6(June 2015)

The folloing is related Chinese contents.

动态结构理论讨论十三

\section{作者 韩雨}

动态结构及其作用是指引起一定现象的原因, 而动态结构及其作用的表现的现象是该原因的结果。原因 是指引起一定现象的现象, 结果是指由于原因的作用, 缘之串联而引起的现象。

由于因果关系及其复杂，有很多关系：

1 先因后果

2 可能还有先果后因（结果在先，原因在后）或因果同时发生

3 其它关系

但是科学不等于真理。科学是为了应用。所以当前这里的因果关系只取先因后果的关系 (随着科学的发 展, 还可能取到其它的关系)。

原因在先, 结果在后（简称先因后果）是因果联系的特点之一, 可原因和结果必须同时具有一定的联系, 即二者的关系属于引起和被引起的关系。

因为动态结构及其作用的表现即有绝对性又有概率性及其它性质。非常复杂。所以当研究一个现象时, 通常取的动态结构及其作用越多（且满足一定的关系）时，由原因推出的结果越准确。 
例: 宏观物体的运动通常用经典力学描述, 就是因为宏观物体的满足一定关系的动态结构及其作用多, 所以表现出经典的运动。而量子力学的用概率描述, 就是因为满足一定关系的动态结构及其作用少。如 果想用经典的方式去描述量子, 则需要取大量的满足一定关系的动态结构及其作用或取更高一级或几级 的动态结构及其作用。一级不够, 就取二级、三级......等。

这就好像, 从湖里打捞一条鱼的质量是很难估计的, 打捞两条鱼的平均质量也是很难估计的, 因为打捞 的鱼的数量很少的情况下, 标准差很大, 这就像量子力学。但是打捞一定数量的鱼的平均质量是容易估 计的, 因为湖里的鱼的质量是正太分布的。例如打捞一万条鱼的平均质量是容易估计的。因为打捞的鱼 的数量很多的情况下, 标准差很小, 有时甚至趋近于零, 这就像经典物理。

由于取动态结构及其作用越多, 数学上可能越复杂, 所以应该用极变数学解决这个复杂的问题。或者一 定级别和常见的动态结构及其作用用特殊的数学关系表达, 然后逐渐发展这些数学关系。

\section{动态结构理论讨论十四}

\section{作者 韩雨}

物质具有粒子性和波动性。其实物质即具有粒子性又具有波动性并不矛盾, 因为物质的粒子性和波动性 都是物质相关动态结构及其作用的的表现 (物质相关动态结构及其作用是指物质本身的动态结构及其作 用及其与其相互作用影响的其它动态结构及其作用)。由于物质的性质是由物质相关动态结构及其作用的 的表现决定的, 所以物质不但有粒子性、波动性, 还有其它性质。物质的质量等所有性质都是由物质相 关动态结构及其作用的的表现决定的。由于几乎所有性质都是动态结构及其作用的表现的结果, 所以几 乎所有性质都是变化的。由于物质的所有性质都是动态结构及其作用的表现的结果, 所以物质的几乎所 有性质都是变化的。

虽然物质的性质是变化的, 但为了方便应用和量化, 即物质的性质符合性质概率定理或叫物质性质概率 定理或性质概率统计定理或物质性质概率统计定理。

性质概率定理是指在一定条件下, 一定量的物质的某方面性质的变化相对不明显, 既有一定的概率规律 或概率统计规律, 即在一定范围内有标准差, 而且该方面性质和标准差的变化都是相对不明显的。

根据性质概率定理, 研究一定量的物质的性质时, 可以取该一定量的物质的统计学指标进行研究、应用 和量化等。

由于质量和能量也是动态结构及其作用的的表现, 所以质量和能量也是变化的。一定量的质量符合性质 概率定理可以叫质量性质概率定理。一定量的能量符合性质概率定理可以叫能量性质概率定理。即一定 量的物质的某方面性质符合性质概率定理，可以称其为该方面性质概率定理。

所以孤立系统的总能量是变化的。孤立系统的总质量也是变化的, 即孤立系统的总的静质量是变化的, 孤立系统的总的动质量也是变化的。所以质量守恒定律、能量守恒定律和质量能量守恒定律都是错误的。

(质量能量守恒定律是指孤立系统的质量和能量的总和是守恒的, 如果质量不守恒了, 那一定是以辐射 的方式放出了能量; 反之亦然。）

质量能量守恒定律是不对的, 应该为在一定条件下的质量能量概率相对守恒定律。因为能量守恒定律是 指在一个封闭 (孤立) 系统的总能量保持不变, 其实在一个封闭 (孤立) 系统的总能量是变化的, 只不 过当在这个封闭 (孤立) 系统的总能量很大的时候, 有很大的概率表现为总能量变化的很小, 即变化的 标准差很小, 而不是没有变化。当在这个封闭 (孤立) 系统的总能量极小的时候, 有很大的概率表现为 总能量变化的很大, 即变化的标准差相对很大, 变化明显。同理, 质量守恒定律和质量能量守恒定律也 是如此。

所以质量守恒定律、能量守恒定律和质量能量守恒定律都是错误的。即应该是质量概率相对守恒定律、 能量概率相对守恒定律和质量能量概率相对守恒定律。

由于质量和能量是相关动态结构及其作用的的表现, 所以在封闭的孤立系统里, 质量和能量也是变化的。 而且可以通过改变封闭的孤立系统里的相关动态结构及其作用而改变封闭的孤立系统里的质量和能量, 即可以不用从外界传递质量或能量也能改变封闭的孤立系统里的质量、能量或质量和能量。所以通过改 
变封闭的孤立系统里的相关动态结构及其作用或封闭的孤立系统里的物质的相关动态结构及其作用或开 放系统的相关动态结构及其作用或开放系统的物质的相关动态结构及其作用而达到改变质量、能量或质 量和能量的变化, 可以解决能源问题、生存空间问题、医疗问题、人的生命永恒问题等 (因为通过改变 相关动态结构及其作用可以再造物质、能量等, 还可以维持与生命相关动态结构及其作的变化在一定范 围, 实现生命的永恒等)。

下面讨论粗楉物理学 (粗楉物理学是指公元 2012 年 6 月以前的物理学)

粗粘物理学认为运动物体的质量随着其运动速度增加而增大。速度的平方除以光速的平方, 这就是将速 度与质量关联起来。

$\mathrm{m}=\mathrm{m}_{0} \div\left(1-\mathrm{V}^{2} \div \mathrm{C}^{2}\right)^{1 / 2}$

所以如果物体做低速运动, 那么其质量的增加就非常小; 然而如果物体运动的速度接近光速, 那么其质 量的增加就非常巨大。从公式 $m=m_{0} \div\left(1-\mathrm{V}^{2} \div \mathrm{C}^{2}\right)^{1 / 2}$ 可以看出, 任何速度增加接近于光速运动的物体的质 量都趋近于无穷大, 因此, 实体物体不可能达到或超过光速。

其实 $m=m_{0} \div\left(1-V^{2} \div C^{2}\right)^{1 / 2}$ 和物体不可能达到或超过光速都是错误的, 甚至可以说 $m=m_{0} \div\left(1-V^{2} \div C^{2}\right)^{1 / 2}$ 和物体不可能达到或超过光速及相对论（包括狭义相对论和广义相对论）都是错误的, 而且 $m=m_{0} \div(1-$ $\left.\mathrm{V}^{2} \div \mathrm{C}^{2}\right)^{1 / 2}$ 和物体不可能达到或超过光速及相对论（包括狭义相对论和广义相对论）不但是错误的, 而且 是糟粕。

因为根据动态结构理论, 物体能达到或超过光速, 甚至远远超过光速。但是物体的质量或能量或质量和 能量不可能达到或趋近于无穷大。物体的很多性质都不可能达到或趋近于无穷大。物体相对于另一物体 的相对速度不同时, 其对于另一物体的相对质量在很多情况下也不同, 是符合动态结构理论的。因为物体 相对于另一物体的相对速度不同时, 它们的相关动态结构及其作用也不同, 所以其对于另一物体的相对 质量在很多情况下也不同。但是它们的相关动态结构及其作用的质量表现有一定的概率可能相对相同 (但 是通常情况下概率很低), 所以其对于另一物体的相对质量在很少情况下也可能相对相同 (不是绝对相 同)。

所以 $m=m_{0} \div\left(1-V^{2} \div C^{2}\right)^{1 / 2}$ 和物体不可能达到或超过光速及相对论（包括狭义相对论和广义相对论）都是 错误的, 是糟粕。

其实一定量的光也是有质量的, 因为物质的质量是变化的, 且符合性质概率定理。因为光的质量很小, 所以一定量的光的质量的性质概率统计指标的均值可能很小, 而且标准差可能相对很大, 所以一定量的 光的质量不容易测量。

根据动态结构理论不但有正质量、负质量, 还有其它质量 (除正质量、负质量以外的质量)。

同理根据动态结构理论不但有正能量、负能量, 还有其它能量 (除正能量、负能量以外的能量)。

同理还有正温度、负温度，还有其它温度（除正温度、负温度以外的温度）。

即根据动态结构理论不但有正性质、负性质，还有其它性质（除正性质、负性质以外的性质）。

\section{动态结构讨论十五}

\section{作者 韩雨}

由于物理规律或物理定律等都是相关动态结构及其作用的表现。所以在不同惯性系中, 物理定律可能不 同，即在不同惯性系中，物理定律的形式可能不同。

由于物理规律或化学规律等自然规律都是相关动态结构及其作用的表现的结果。所以在不同惯性系中, 物理定律、化学定律等自然规律可能不同, 即在不同惯性系中, 物理定律、化学定律等自然规律的形式 可能不同。

因为不同的惯性系可能意味着相关动态结构及其作用的不同, 而传统的粗糙的物理学并未意思到物理规 律或化学规律等是相关动态结构及其作用的表现的结果。所以传统的粗粘的物理学错误的认为: “在所有 惯性系中，物理定律的形式都相同。”

传统的粗粘的物理学之所以未发现“在不同惯性系中, 物理定律可能不同”, 是因为传统的粗粘的物理学 所研究的对象都是相关动态结构及其作用的宏观表现, 而相关动态结构及其作用的宏观表现有很大概率 
表现为宏观物理定律在不同惯性系中不变。

例如: 研究宏观物体的运动, 通常用经典力学, 而且并未发现经典力学的缺点。当研究微观粒子时, 就 会发现经典力学的错误, 而采用量子力学。同理, 在继续往深研究, 就会发现, 经典力学、经典电磁学、 经典统计力学、经典光学、量子力学、相对论等传统的粗糙的物理学的错误, 而采用动态结构理论。

当然, 随着研究的继续深入, 会发现低级动态结构的不适用性, 而采用高级的动态结构, 当研究无限深 入, 就会得到真理——极变理论, 即采用极变理论 (即采用真理)。

即, 一级动态结构不适用, 采用二级动态结构。二级动态结构不适用, 采用三级动态结构......n 级动态 结构不适用, 采用 $n+1$ 级动态结构......无穷大级动态结构——真理的极变理论。所以动态结构理论是可 发展的科学理论, 是不会被取代的科学理论, 是真正的科学理论。所以应该大力发展动态结构理论。 根据动态结构理论, 在惯性系中, 无论光源或观察者是否运动或静止, 真空中的光速都有可能变化。 根据非单调运动或非单调变化, 在惯性系中, 即使观察者静止, 在一定范围内, 真空中的光速都是变化 的。其实在惯性系中, 相对于静止的观察者, 真空中的光速也是变化的。之所以, 人们认为“在惯性系中, 相对于静止的观察者, 真空中的光速不变” 是因为真空中的光速变化不明显, 而人们只注意到了真空中的 光速的宏观或明显的表现, 而未注意到光速的不明显的变化。

下面讨论传统的粗粘的物理学。

在传统的粗糙的物理学中, 只考虑光速的宏观或明显的表现而不考虑光速的不明显的变化, 即认为“在惯 性系中, 相对于静止的观察者, 真空中的光速不变”。

其实, 即便对于传统的粗糙的物理学, 在惯性系中, 相对于运动的观察者, 真空中的光速也是变化的。 例; 在惯性系中, 真空中的静止的光源向观察者发出一束光, 观察者向光源移动的速度为 $\mathrm{V}$, 真空中的 光速为 $\mathrm{C}$ ，则光相对于观察者的速度为 $\mathrm{C}+\mathrm{V}$ 。

例: 在惯性系中, 真空中有一个向静止的观察者移动的光源, 光源的速度为 $\mathrm{V}$, 光源向静止的观察者发 出一束光, 真空中的光速为 $\mathrm{C}$, 则光相对于观察者的速度仍约为 $\mathrm{C}$ 。因为发生了能量阻碍作用, 根据能 量阻碍方程得光相对于观察者的速度仍约为 $\mathrm{C}$ 。

\section{动态结果理论讨论十六}

\section{作者 韩雨}

波是相关动态结构及其作用的表现。可以大概简单粗略的分为三种情况:

1.波是相关动态结构及其作用的表现的本体表现或体现。

2.波是相关动态结构及其作用的表现的传递或传播。

3.波是相关动态结构及其作用的表现的其它形式。

目前传统的物理学中的机械波、电磁波、光波等都可用相关动态结构及其作用的表现的传递或传播解释。 所以当前可以认为机械波、电磁波、光波等所有波都是相关动态结构及其作用的表现的传递或传播（其 实, 这些波还有其它两种波的表现及其它动态结构及其作用的表现, 但是为了实现系统的对接, 可以把 这些波简单粗略的认为是相关动态结构及其作用的表现的传递或传播, 用这种方法足以解决传统的物理 学中的机械波、电磁波、光波等所有波)。

根据波是相关动态结构及其作用的表现的传递或传播讨论传统的物理学中的机械波、电磁波、光波等: 因为波是相关动态结构及其作用的表现的传递或传播。所以电磁波、引力波、光波、机械波等所有波都 必须依靠相关动态结构及其作用的表现才能传播。即波只能在相关动态结构及其作用的表现中传播。因 为波只能在相关动态结构及其作用的表现中传播, 所以波速与相关动态结构及其作用的表现有关。波速 不但与相关动态结构及其作用的表现有关, 而且与波源的性质也有关。因为波源或波源的性质的本质是 相关动态结构及其作用的表现, 而波的传播是波源或波源的性质的相关动态结构及其作用与传播媒介的 相关动态结构及其作用的相互作用的表现。所以波速与波源的性质有关。

传统物理学认为除了电磁波和引力波能够在真空中传播外, 大部分波如机械波只能在介质中传播。其实 传统物理学中的真空并不是什么都没有, 而是有相关动态结构及其作用的表现。

传统物理学认为机械波只能在介质中传播, 其实传统物理学中的介质就是相关动态结构及其作用的表现。 
电磁波和引力波之所以能够在真空中传播，也是因为真空中的相关动态结构及其作用的表现。

\section{动态结构理论讨论十七}

\section{作者 韩雨}

精神是不同于物质的, 关于精神的时间也是不同于物质的时间的。关于精神的许多东西都不同于物质的 东西。精神是极其复杂的。只有当动态结构理论或极变理论发展到一定程度, 才能有效的研究精神与精 神以外的物质世界等的关系。所以, 当前的物理学、化学等自然科学只研究物质世界。

在讨论中，如果未特殊提到精神，则表示讨论的只是物质世界。

物质的时间和空间与物质的其它方面相比也是非常复杂的。真正的或真理的时间不能用长短快慢等现在 的物理量简单精确的描述。真正的或真理的空间也不能用现在的物理量简单精确的描述。只有当动态结 构理论或极变理论发展到一定程度, 才能有效的研究真正的或真理的时间与空间。但是, 在当前物理学、 化学等自然科学中经常面对时间和空间的问题。为了使物理学、化学等自然科学更好的发展, 下面规定 时间和空间的概念 (不是真正的或真理的时间和空间的概念, 而是为了自然科学更好的发展而规定的概 念)。

时间是抽象概念, 表达事物的生灭排列。其内涵是无尽永前, 其外延是一切事件过程长短和发生顺序的 度量。“无尽”指时间没有起始和终结, “永前”指时间的增量总是正数。同时, 时间也不可能被暂停和逆 转。

空间表达物件的范围。内涵是无界永在, 外延是一切物件占位大小和相对位置的度量。“无界”指空间没 有界限, “永在”指空间永现于当前时刻。

在讨论中如未特殊提及时间和空间的概念，就指上面的概念。

由于相关动态结构及其作用的表现书写复杂, 所以在讨论中把相关动态结构及其作用和相关动态结构及 其作用的表现等综合起来简称为广义动态结构, 或称为动表结构。

波是相关动态结构及其作用的表现。可以大概简单粗略的分为三种情况, 为方便讨论把三种情况命名。

1.波是相关动态结构及其作用的表现的本体表现或体现的这类波称为本体波。

2.波是相关动态结构及其作用的表现的传递或传播的这类波称为传递波或传播波或传波。

3.波是相关动态结构及其作用的表现的其它形式的这类波称为其它波。

由于动表结构既有超距作用又有近距作用, 还有其它作用。所以动表结构及其复杂, 为了实现系统的对 接，只考虑动表结构的起决定作用的主要的作用。

由于电磁力、引力等各种当前的物理学中的力的本质是动表结构, 所以电磁力、引力等各种当前的物理 学中的力既有超距作用又有近距作用, 还有其它作用形式。但电磁力、引力等各种当前的物理学中的力 的主要作用形式是近距作用。这种近距作用是通过传递波的形式发挥主要作用的。

波的属性大概可以分为五种:

1 性质和形式

2 结构

3 方向

4 量或数量和能量的大小

5 其它属性

例: 引力波与电磁波的性质和形式以及结构等都是不同的。

例：正电荷的传递波与负电荷的传递波性质和形式相同，但方向不同。

为了方便理解, 下面打几个比喻:

例：引力波的传递波就好像是漩浴一样的场，使其向漩浴的中心移动。一个引力波的传递波使另一个引 力波的传递波向其中心传播和运动。而引力波的传递波又使物质向其中心运动。

例：正电荷的传递波与负电荷的传递波的方向不同，就好像正电荷的传递波是顺时针方向的，而负电荷 的传递波是逆时针方向的。所以同种电荷之间的传递波是互斥的，不同电荷之间的传递波是互相汇聚传 播和运动的。而电荷及其传递波又有近距作用。所以才会有同种电荷互相排斥, 异种电荷互相吸引的现 
象。

动态结构理论讨论十七的应用

\section{作者 韩雨}

根据非单调变化，可知不存在无穷大的能量和质量等物理量。即使是黑洞的质量也不是无穷大。 随着对物质世界研究的深入, 物质世界可能即存在远距作用又存在近距作用, 继续深入, 可能即不存在 远距作用也不存在近距作用。但是为了当前物理学的有效发展, 可认为引力的作用形式主要是动表结构 的传递波。

下面应用传递波的理论讨论质量。

传统物理学认为任何物体都具有吸引其他物体的性质, 引力质量是物体这种性质的量度。所以引力质量 主要是通过传递波的形式得以表现。物质的动表结构产生传递波，使物质的引力质量得以表现。当物质 聚集在一起时，质量（这里指稳定的质量，即质量的大小波动不明显）开始变大，即引力的传递波的作 用形式增强, 当物质聚集到一定程度时, 由于非单调变化, 再继续增加聚集物质, 质量可能开始降低, 即引力的传递波的作用形式减弱。

由于引力质量主要是通过传递波的形式得以表现出引力, 而传递波是有方向性的。所以与引力质量传递 波的方向相反的传递波的表现主要是斥力或负引力, 而产生这种负引力的传递波的物质可以用负质量度 量。

为了方便理解, 下面打几个比喻:

例: 正质量的引力波的传递波就好像是漩浴一样的场 (立体的漩浴), 使其向漩浴的中心移动。一个引力 波的传递波使另一个引力波的传递波向其中心传播和运动。而引力波的传递波又使物质向其中心运动。 而负质量的负引力波的传递波就好像是反向漩浴一样的场, 使其由漩浴的中心向外运动。一个引力波的 传递波使另一个引力波的传递波由中心向外运动。所以负质量产生负引力。与正质量一样, 负质量也不 可能无穷大，原因同正质量一样。当引力波的传递波与负引力波的传递波相互作用时或叠加时（与传统 物理学中的波的叠加类似), 两种传递波的力的表现相互作用或叠加, 正引力和负引力相互抵消的部分变 成其它形式的动表结构, 这种动表结构可以表现出波的三种形式的任意一种或几种, 即本体波、传递波 和其它波。但很多情况下, 这种动表结构通常也以传递波的形式表现，或者说这种动表结构被辐射出去。 因为传统物理学中的辐射的本质是传递波，所以动表结构以传递波的形式表现或以辐射的形式表现其本 质是一样的。这种动表结构也会以传递波的形式相互作用或叠加。叠加到一定程度时, 可以被相对容易 的发现。动表结构以传递波的形式表现还可以分为几种情况:

1、快速形式: 当引力波的传递波与负引力波的传递波相互作用时或叠加时, 快速产生相对明显的动表结 构。

2、慢速形式: 当引力波的传递波与负引力波的传递波相互作用时或叠加时, 产生相对明显的动表结构的 时间很长。

3、其它形式：除快速形式和慢速形式以外的其它形式。

例：黑洞的质量达到一定条件时，黑洞再继续吸收质量，则该黑洞可以产生负引力的传递波，负引力的 传递波与引力的传递波相互叠加，抵消一部分黑洞的引力，也相当于黑洞产生了负质量，抵消了一部分 正质量, 所以黑洞的质量不会无穷大。黑洞的质量达到一定条件时，再继续吸收质量，黑洞的质量会下 降。当黑洞的负引力的传递波与引力的传递波相互叠加, 抵消一部分黑洞的引力时, 也会产生其它形式 的动表结构, 通常都会辐射出去。所以黑洞会发出一定的辐射是很正常的。

\section{动态结构讨论十七补充}

\section{作者 韩雨}

因为物质、能量及其相互作用等都是相关动态结构及其作用的表现。所以波的叠加及能量守恒等也是相 关动态结构及其作用的表现。

下面讨论光的叠加问题（其它形式的波可用相同或类似的方法讨论，所以下面只讨论光波）: 传统物理学认为, 光波强度为 $I_{1}$ 的光波, 与光波强度为 $I_{2}$ 的光波发生干涉时, 其干涉后的总光强为 $\mathrm{I}$, 
$\mathrm{I}=\mathrm{I}_{1}+\mathrm{I}_{2}+2\left(\mathrm{I}_{1} \times \mathrm{I}_{2}\right)^{1 / 2} \times \cos \delta$

当 $\cos \delta=1$ 时, 发生相长干涉

此时, $I=I_{1}+I_{2}+2\left(I_{1} \times I_{2}\right)^{1 / 2}>I_{1}+I_{2}$.

之所以会出现这样的情况, 是因为当 $I_{1}$ 和 $I_{2}$ ( $I_{1}$ 和 $I_{2}$ 不是特别的大) 在空间中传播时, 其实不但有 $I_{1}$ 和 $\mathrm{I}_{2}$ 沿着动表结构传播, 还有一不明显的能量扰动沿动表结构传播, 这个不明显的能量称为 $\mathrm{I}_{\mathrm{r}}$ (波阻抗的本 质可能就是 $I_{r}$, , 这里分别称为 $I_{1 r}$ 和 $I_{2 r}$ 当 $I_{1}$ 和 $I_{2}$ 发生干涉时, $I_{1 r}$ 和 $I_{2 r}$ 发生明显的改变, 一部分能量变成 $I$, 所以 $I=I_{1}+I_{2}+2\left(I_{1} \times I_{2}\right)^{1 / 2}>I_{1}+I_{2}$ 。 所以符合能量概率相对守恒定律。或者说能量概率相对守恒中发生 相对守恒的概率很高，甚至趋近于 $100 \%$.

当 $I_{1}$ 和 $I_{2}$ 特别大时, 即足够大时或达到一定条件时, 此时 $I_{1}$ 和 $I_{2}$ 发生干涉时, 不但 $I_{1 r}$ 和 $I_{2 r}$ 发生明显的 改变, 一部分能量变成 I, 而且由于动表结构发生了明显改变, I 会有更大的能量表现, 因为动表结构的 明显改变可以导致能量的明显改变或巨变或突变, 因为能量的本质是动表结构。此时, 不但

$I>I_{1}+I_{2}$, 而且也有可能发生 $I>I_{1}+I_{2}+2\left(I_{1} \times I_{2}\right)^{1 / 2}$ 。甚至有可能发生 $I<I_{1}+I_{2}+2\left(I_{1} \times I_{2}\right)^{1 / 2}$ 或 $I<I_{1}+I_{2}$ 或 $I<I_{1}+I_{2}-2\left(I_{1} \times I_{2}\right)^{1 / 2}$ 等多种情况（此时需要对动表结构做具体的分析）。此时能量可能明显的不守 恒，或则说能量概率相对守恒中发生相对守恒的概率很低，甚至趋近于 0 .

当 $\cos \delta=-1$ 时, 发生相消干涉

$\mathrm{I}=\mathrm{I}_{1}+\mathrm{I}_{2}-2\left(\mathrm{I}_{1} \times \mathrm{I}_{2}\right)^{1 / 2}<\mathrm{I}_{1}+\mathrm{I}_{2}$

同理当 $I_{1}$ 和 $I_{2}$ 不是特别的大时, 有一部分能量直接或间接转化为其它形式的能量, 例如 $I_{r}$ 、热能、磁能 等, 所以符合能量概率相对守恒定律。或者说能量概率相对守恒中发生相对守恒的概率很高, 甚至趋近 于 $100 \%$.

同理当 $I_{1}$ 和 $I_{2}$ 特别大时, 即足够大时或达到一定条件时, 此时 $I_{1}$ 和 $I_{2}$ 发生干涉时, 由于动表结构发生了 明显改变, 会发生巨变, 有可能 $\mathrm{I}<\mathrm{I}_{1}+\mathrm{I}_{2}-2\left(\mathrm{I}_{1} \times \mathrm{I}_{2}\right)^{1 / 2}$ 或 $\mathrm{I}>\mathrm{I}_{1}+\mathrm{I}_{2}+2\left(\mathrm{I}_{1} \times \mathrm{I}_{2}\right)^{1 / 2}$ 等多种情况（此时需 要对动表结构做具体的分析)。此时能量可能明显的不守恒, 或则说能量概率相对守恒中发生相对守恒的 概率很低，甚至趋近于 0 .

\section{极变理论与动态结构理论}

\section{作者 韩雨}

极变理论是指事物的某方面在系统内达到极限, 系统不在适合该事物继续向原方向发展, 如果系统不发 生变化，事物在该方面会发生质变或向相反的方向变化而很难或几乎不能或不可能保持原来的状态。

极变理论实际上是说明了自然界真正的规律、原理和本质。极变理论实际上说明了事物发展的真正的规 律、原理和本质。极变理论实际上是指事物是按照事物真正的发展规律、发展方向、发展方式等发展的。 极变理论实际上是指事物是按照事物真正的、实际的发展规律、发展方向、发展方式等发展的。极变理 论实际上是事物及其发展的本质。所以极变理论是真理。从狭义上说, 极变理论就是事物及其发展的真 理。从广义上说，极变理论就是事物及其发展。极变理论实际上说明了事物是按照其真正的、实际的发 展规律、发展方向、发展方式等运行的。

例：对于一个真正的客观事实：事物 $\mathrm{A}$ 变化到事物 $\mathrm{B}$ ，再变化到事物 $\mathrm{C}$ 。

极变理论就说明了事物 $\mathrm{A}$ 变化到事物 $\mathrm{B}$, 再变化到事物 $\mathrm{C}$ 。

从狭义上说, 极变理论就是事物 $\mathrm{A}$ 变化到事物 $\mathrm{B}$, 再变化到事物 $\mathrm{C}$ 的真理。从广义上说, 事物 $\mathrm{A}$ 变化到 事物 B, 再变化到事物 C 就属于极变理论。

\section{极变理论的解释}

\section{作者 韩雨}

极变理论是指事物的某方面在系统内达到极限, 系统不在适合该事物继续向原方向发展, 如果系统不发 生变化，事物在该方面会发生质变或向相反的方向变化而很难或几乎不能或不可能保持原来的状态。 极变理论实际上是说明了自然界真正的规律、原理和本质。极变理论实际上说明了事物发展的真正的规 律、原理和本质。极变理论实际上是指事物是按照事物真正的发展规律、发展方向、发展方式等发展的。 极变理论实际上是指事物是按照事物真正的、实际的发展规律、发展方向、发展方式等发展的。极变理 
论实际上是事物及其发展的本质。所以极变理论是真理。从狭义上说, 极变理论就是事物及其发展的真 理。从广义上说, 极变理论就是事物及其发展。极变理论实际上说明了事物是按照其真正的、实际的发 展规律、发展方向、发展方式等运行的。

例：对于一个真正的客观事实：事物 $\mathrm{A}$ 变化到事物 $\mathrm{B}$, 再变化到事物 $\mathrm{C}$ 。

极变理论就说明了事物 $\mathrm{A}$ 变化到事物 $\mathrm{B}$, 再变化到事物 $\mathrm{C}$ 。

从狭义上说, 极变理论就是事物 $\mathrm{A}$ 变化到事物 $\mathrm{B}$, 再变化到事物 $\mathrm{C}$ 的真理。从广义上说, 事物 $\mathrm{A}$ 变化到 事物 B，再变化到事物 C 就属于极变理论。

\section{动态结构理论的解释}

\section{作者 韩雨}

因为事物是发展变化的。事物发展变化的原因也是变化的。因为事物发展变化的原因是发展变化的, 所 以事物发展变化的原因是动态的 (这里的动态指发展变化)。动态结构理论中的动态指发展变化, 结构是 指原因。所以动态结构理论是指发展变化的原因理论; 是指事物发展变化的原因是发展变化的理论; 是 指事物发展变化的过程和结果的原因。动态结构理论也可以叫动态原因理论或发展变化原因理论或变化 原因理论。动态结构理论发展到极致就是真理——极变理论。

因为科学中, 常用结构决定功能和性质, 所以把动态原因理论叫动态结构理论。这里的结构指的是原因。

\section{动态结构理论讨论十八}

\section{作者 韩雨}

因为引力波、电磁波、光波等都是动态结构及其作用的表现, 所以引力波、电磁波、光波等之间有相互 作用。即电磁场、引力场、光场等之间有相互作用。引力、库仑力、安培力、洛仑兹力等电磁力及其它 各种力等都是动态结构及其作用的表现, 所以引力、库仑力、安培力、洛仑兹力等电磁力及其它各种力 等之间有相互作用。力可以看作是动态结构及其作用的表现。

\section{动态结构讨论十九}

\section{作者 韩雨}

物质是动态结构及其作用的表现。无论是宏观物质还是微观物质都与外界的动态结构及其作用相互作用, 其实物质是与外界的动态结构及其作用相互作用维持其稳定性的（这种稳定性主要是相对的动态的稳定 性)。物质与外界的动态结构及其作用相互作用的方式有很多种。例如: 物质的动态结构及其作用于外界 的动态结构及其作用相互交换, 维持动态平衡。

例如: 电子、中子、质子等它们都与外界的动态结构进行交换, 维持其自身的稳定性。如果不与外界的 动态结构进行交换, 则它们会发生变化, 电子可能不再是电子了, 失去原有的性质。同样中子和质子也 失去原有的性质，不再是中子和质子。

其实, 物质及其性质都是动态结构及其作用的表现。无论是宏观物质还是微观物质的性质或属性都与外 界的动态结构及其作用相互作用, 其实物质的性质或属性是与外界的动态结构及其作用相互作用维持其 稳定性的 (这种稳定性主要是相对的动态的稳定性)。物质的性质或属性与外界的动态结构及其作用相互 作用的方式有很多种。例如: 物质的性质或属性的动态结构及其作用于外界的动态结构及其作用相互交 换, 维持动态平衡。

例如: 电阻阻碍导电的性质就是电阻阻碍导电的性质的动态结构于外界的动态结构相互作用维持电阻的 性质的稳定性的。如果电阻不与周围的动态结构进行交换，电阻的性质可能很快遭到破坏，电阻的性质 将明显改变。

其实自然界的物事都是与外界的动态结构及其作用相互作用的。从而维持其稳定性（既有绝对稳定性、 又有相对稳定性，还有其它稳定性）。

\section{能量阻碍方程的动态结构理论讨论}

\section{作者 韩雨}

由于物质和能量等都是动态结构及其作用的表现, 所以能量阻碍效应也符合动态结构理论。导致能量阻 碍效应主要有 
1 施加能量的客体的动态结构及其作用决定了该客体施加能量的多少、形式、能力等

2 接受能量的客体的动态结构及其作用决定了该客体对能量接受的多少、形式、能力等

3 施加能量的客体和接受能量的客体的动态结构及其作用之间的相互作用对能量阻碍也有影响

4 施加能量的客体和接受能量的客体的动态结构及其作用会受到周围的的动态结构及其作用的影响

5 其它方面

\section{相似定理}

\section{作者 韩雨}

物质、能量及物质和能量的运动和变化等都是动态结构及其作用的表现。所以物质、能量及其运动和变 化等有一定的相似性。

相似定理是指表现相似（或相同）互相排斥 (或产生互相排斥的趋势), 相似程度越高, 排斥（或排斥的 趋势）越明显。

即在一定条件下, 表现相似 (或相同) 则有一定概率互相排斥（或有一定概率产生互相排斥的趋势），相 似程度越高, 排斥 (或排斥的趋势) 的概率越大, 即越明显。相似定理又可叫相似结构作用定理、相似 作用定理或相似排斥定理。

物质、能量及物质和能量的运动和变化等相似, 则有互相排斥的趋势, 相似程度越高, 排斥 (或排斥的 趋势）越明显。

即物质、能量及物质和能量的运动和变化等相似, 则有一定概率产生互相排斥的趋势, 相似程度越高, 排斥（或排斥的趋势）的概率越大，即越明显。

例如; 热量的由高向低的传导。压强的传导。离子由高浓度向低浓度的扩散等等，都遵守相似定理。

例如, 组成 $\mathrm{Na}$ 原子的动态结构及其作用的表现有一定相似性。组成 $\mathrm{K}$ 原子的动态结构及其作用的表现 有一定相似性。组成质子的动态结构及其作用的表现有一定相似性。组成中子的动态结构及其作用的表 现有一定相似性。组成电子的动态结构及其作用的表现有一定相似性。

同一种形式的能量的动态结构及其作用的表现有一定相似性。同一种形式的大小相同的能量的动态结构 及其作用的表现有一定相似性。大小相同的能量的动态结构及其作用的表现有一定相似性。

动能的动态结构及其作用的表现有一定相似性。电能的动态结构及其作用的表现有一定相似性。热能的 的动态结构及其作用的表现有一定相似性。

100 摄氏度的水的动态结构及其作用的表现有一定相似性。5 摄氏度的水的动态结构及其作用的表现有一 定相似性。

\section{应用一元论的动态结构理论基础}

\section{作者 韩雨}

多元论主张世界是由多种本原构成的哲学学说, 但其在科学的量化、应用等方面复杂。一元论 (Monism) 是认为世界只有一个本原的哲学学说, 一元论的观点是错误的。同样, 唯物主义也是错误的。唯物主义 一元论也是错误的。

现实应用一元论是指把世界简化、近似、抽象的看成为只有一个本原的科学的哲学学说, 简称应用一元 论。

现实应用唯物主义其实也是把世界简化、近似、抽象的看成为由物质组成。

现实应用唯物主义一元论是两者的结合。

由于科学不是真理。科学要求能够很好的应用, 所以科学是对规律的一种简化、近似、抽象等。自然科 学是对自然规律的一种简化、近似、抽象等。

通常情况下, 应用一元论或唯物主义一元论可以方便的对规律进行简化、近似、抽象等。所以科学研究 时, 经常应用一元论。

通常情况下，一元论之所以能够对自然规律行简化、近似、抽象等，是因为动态结构及其作用的综合统 计表现导致的。

因为在一定条件下, 大量的动态结构及其作用的综合统计表现出了一些客体的相似性, 自然科学常将其 
称为共性，经常把具有相似性的客体看成为同一种客体。

例: 宏观的两个不同金属球，在研究质量时，不考虑它们化学上的差别，认为它们的质量是同一种表现。 只要这两个球的质量相同, 它们所受的重力是一样的。不考虑它们化学上的差异。

例; 世界上的狮子都是不同的, 但不考虑其它不同的方面, 只研究它们的相识性, 所以把它们统称为狮 子。

杨树和柳树是不同的，但只考虑它们都是树的性质时，把它们统称为树。

例; 每个电子都是不同的, 但考虑它们的相识性时, 把它们统称为电子。同理, 质子、中子等也是如此。 其实电子质子、中子、微观或宏观物质等客体之所以有相似性, 是因为在一定条件下, 大量的动态结构 及其作用的综合统计表现出了一些客体的相似性。

例; 每个电子都是在一定条件下, 大量的动态结构及其作用的综合统计表现, 由于动态结构及其作用的 量很大，导致了每个电子之间在某些方面的性质十分相似，当动态结构及其作用的量达到一定条件时， 可以简化、近似、抽象的认为每个电子之间是无差异的。

这类似于, 在湖里捞鱼, 每天捞鱼的数很少时, 则每天捞鱼的总质量是有差异的, 是不同的。如果每天 捞鱼的数量特别多时, 每天捞鱼的总质量是相近的 (因为每天捞鱼的质量是正太分布的)。当每天捞鱼的 数量大到一定程度时，可以简化、近似、抽象的认为每天捞鱼的总质量是相同的。

这就类似于在一个正太分布的群体中, 选取 $\mathrm{n}$ 个个体, 研究这 $\mathrm{n}$ 个个体的均值。当 $\mathrm{n}$ 越大时, 它们的标 准差越小，当 $\mathrm{n}$ 大到一定程度时，标准差趋近于 0 .

当 $\mathrm{n}$ 小时，曲线分布形状“扁平”，当 $\mathrm{n}$ 逐渐增大时，曲线分布形状“集中”，当 $\mathrm{n}$ 大到一定程度时，曲线 类似与 $\delta$ 函数。

\section{概率相对守恒定律}

\section{作者 韩雨}

概率相对守恒定律是指一定条件下, 自然界的表现或自然的表现或表现的守恒为相对守恒, 即在一定范 围内有标准差, 而且表现和标准差都是变化的。质量能量相对守恒定律是概率相对守恒定律的一种。

例如, 质量、能量等都是表现。

例如, 质量能量守恒定律是不对的, 应该为在一定条件下的质量能量相对守恒定律。因为能量守恒定律 是指在一个封闭 (孤立) 系统的总能量保持不变, 其实在一个封闭 (孤立) 系统的总能量是变化的, 只 不过当在这个封闭 (孤立) 系统的总能量很大的时候, 有很大的概率表现为总能量变化的很小, 即变化 的标准差很小, 而不是没有变化。当在这个封闭 (孤立) 系统的总能量极小的时候, 有很大的概率表现 为总能量变化的很大, 即变化的标准差相对很大, 变化明显。同理, 质量守恒定律也是如此。 